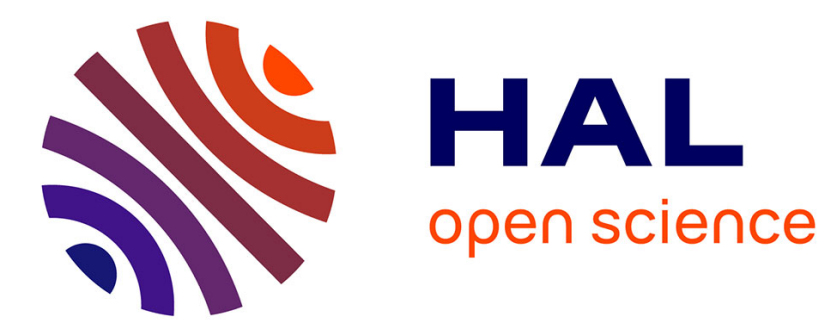

\title{
Thermoelectric properties of epitaxial TbAs:InGaAs nanocomposites
}

Laura E. Clinger, Gilles Pernot, Trevor E. Buehl, Peter G. Burke, Arthur C. Gossard, Christopher J. Palmstrøm, Ali Shakouri, Joshua M. O. Zide

\section{- To cite this version:}

Laura E. Clinger, Gilles Pernot, Trevor E. Buehl, Peter G. Burke, Arthur C. Gossard, et al.. Thermoelectric properties of epitaxial TbAs:InGaAs nanocomposites. Journal of Applied Physics, 2012, 111 (9), pp.094312. 10.1063/1.4711095 . hal-01535967

\section{HAL Id: hal-01535967 \\ https://hal.univ-lorraine.fr/hal-01535967}

Submitted on 9 Jun 2017

HAL is a multi-disciplinary open access archive for the deposit and dissemination of scientific research documents, whether they are published or not. The documents may come from teaching and research institutions in France or abroad, or from public or private research centers.
L'archive ouverte pluridisciplinaire HAL, est destinée au dépôt et à la diffusion de documents scientifiques de niveau recherche, publiés ou non, émanant des établissements d'enseignement et de recherche français ou étrangers, des laboratoires publics ou privés.

\section{(1) (1) $\$$}

Distributed under a Creative Commons Attribution - NonCommercial - NoDerivatives| 4.0 


\title{
Thermoelectric properties of epitaxial TbAs:InGaAs nanocomposites
}

\author{
Laura E. Clinger, ${ }^{1}$ Gilles Pernot, ${ }^{2}$ Trevor E. Buehl, ${ }^{3}$ Peter G. Burke, ${ }^{3}$ Arthur C. Gossard, ${ }^{3}$ \\ Christopher J. Palmstrøm, ${ }^{3,4}$ Ali Shakouri, ${ }^{2}$ and Joshua M. O. Zide ${ }^{1, a)}$ \\ ${ }^{1}$ Materials Science and Engineering Department, University of Delaware, Newark, Delaware 19716, USA \\ ${ }^{2}$ Electrical Engineering Department, University of California, Santa Cruz, California 95064, USA \\ ${ }^{3}$ Materials Department, University of California, Santa Barbara, California 93106, USA \\ ${ }^{4}$ Electrical and Computer Engineering Department, University of California, Santa Barbara, \\ California 93106, USA
}

(Received 10 January 2012; accepted 11 April 2012; published online 9 May 2012)

\begin{abstract}
InGaAs lattice-matched to InP was grown by molecular beam epitaxy with randomly distributed TbAs nanoparticles for thermoelectric power generation applications. TbAs:InGaAs is expected to have a large thermoelectric figure of merit, ZT, particularly at high temperatures, owing to energy band alignment between the nanoparticles and their surrounding matrix. Here, the room temperature thermoelectric properties were measured as a function of TbAs concentration, revealing a maximum thermoelectric power factor of $2.38 \mathrm{~W} / \mathrm{mK}^{2}$ and $\mathrm{ZT}$ of 0.19 with $0.2 \%$ TbAs. Trends in the thermoelectric properties closely resemble those found in comparable ErAs:InGaAs nanocomposite materials. However, nanoparticles were not observed by scanning transmission electron microscopy in the highest ZT TbAs:InGaAs sample, unlike the highest ZT ErAs:InGaAs sample $(0.2 \%$ ErAs) and two higher concentration TbAs:InGaAs samples examined. Consistent with expectations concerning the positioning of the Fermi level in these materials, ZT was enhanced by TbAs incorporation largely due to a high Seebeck coefficient, whereas ErAs provided InGaAs with higher conductivity but a lower Seebeck coefficient than that of TbAs:InGaAs. Thermal conductivity was reduced significantly from that of intrinsic thin-film InGaAs only with TbAs concentrations greater than $\sim 1.7 \%$. C 2012 American Institute of Physics. [http://dx.doi.org/10.1063/1.4711095]
\end{abstract}

\section{INTRODUCTION}

A thermoelectric (TE) device is able to generate power from a temperature gradient or generate a temperature gradient from power input. TE devices can be useful for localized heating or cooling, as in a Peltier cooler, or for generating electricity from technologies where waste heat is created, such as in power plants or in various means of transportation. The viability of TE devices rests primarily on the efficiency of the $\mathrm{n}$ - and p-type materials comprising the device, which is governed by both the Carnot efficiency and the thermoelectric figure of merit, $\mathrm{ZT}=\mathrm{S}^{2} \sigma \mathrm{T} / \kappa$, where $\mathrm{S}$ is the Seebeck coefficient, $\sigma$ is electrical conductivity, $\mathrm{T}$ is temperature, and $\kappa$ is thermal conductivity. ${ }^{1}$ The majority of TE devices currently on the market utilize bulk semiconducting materials which are alloyed to reduce thermal conductivity through increased phonon scattering, yielding ZT of $\sim 1$ near room temperature and efficiencies which are suitable for certain niche applications. In order for the TE devices to enter the wider power generation playing field, the efficiency of TE materials over a wide temperature range must increase dramatically. ${ }^{2}$

Nearly all recent TE materials research has centered on utilizing nano-scale structures within semiconductors to lower thermal conductivity below the alloy limit with hopes of simultaneously increasing (or at least minimally sacrificing) the intrinsically coupled thermoelectric power factor $\left(\mathrm{S}^{2} \sigma\right){ }^{2,3}$ One such set of materials involves co-doping epitaxial III-V semiconductors like InGa(Al)As and InGa(Al)Sb

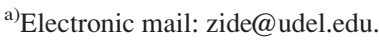

with the rare earth metal Er, which, at levels exceeding the solid solubility limit in the semiconductors, precipitates into randomly distributed semimetallic Er-V nanoparticles. The nanoparticles serve to reduce thermal conductivity (through phonon scattering), increase Seebeck coefficient (through energy-dependent electron scattering), and increase electrical conductivity (through nanoparticle donation of electrons). ${ }^{3-5}$ Progress in the development of this material system, which targets not only heat transport but also seeks to optimize electronic properties, has thus far led to an n-type ZT of 1.33 at $800 \mathrm{~K}$ for 0.6 at. $\%$ ErAs in InGaAlAs. ${ }^{5}$

TbAs is expected to provide certain benefits over ErAs and $\mathrm{ErSb}$, due primarily to its larger lattice constant which is anticipated to more favorably alter electronic properties while providing similar reduction in thermal conductivity. ${ }^{6}$ It has been observed both theoretically and experimentally that strain on the nanoparticles brought about by their latticemismatch to the matrix may be the major factor in the positioning of the Fermi level within the semiconductor's band gap. ${ }^{5,7}$ Indeed, tensile strain on the nanoparticles causes the Fermi level to be shifted up from a universal donor level while compressive strain causes it to be shifted down. Although we believe this trend to be true, it is very difficult to calculate the exact energy levels theoretically; ${ }^{8}$ therefore, experimental evidence is needed. The tensile strain on ErAs nanoparticles in InGaAs and InGaAlAs has been theorized and experimentally observed to pin the Fermi level of these matrices above their conduction bands. ${ }^{5,7}$ Alternatively, TbAs, which is less strained than ErAs in InGaAs with $\sim 0.9 \%$ tensile mismatch, is expected to pin the Fermi level 
below the conduction band. The Seebeck coefficient of TbAs:InGaAs should then be improved since this quantity is theoretically dependent on the moment of the energydependent differential conductivity about the Fermi level, which would increase relative to ErAs-containing materials. ${ }^{2,6,9}$

\section{MATERIAL GROWTH AND PROPERTIES}

In this paper, the room temperature thermoelectric properties of TbAs:InGaAs are explored as a function of rare earth concentration, and a percentage of TbAs which maximizes room temperature thermoelectric power factor and figure of merit is determined. All the samples were grown in an OSEMI NextGEN molecular beam epitaxy (MBE) system by co-depositing Tb during the growth of $\operatorname{In}_{0.53} \mathrm{Ga}_{0.47} \mathrm{As}$ (lattice-matched to the semiinsulating (100) InP substrates). All but 3 of the 20 samples were grown with a $100 \mathrm{~nm} \mathrm{In}_{0.53} \mathrm{Ga}_{0.47} \mathrm{As}$ buffer layer in an attempt to enable post-growth comparison of the compositions of the buffer and active layers using $\mathrm{x}$-ray diffraction (XRD). The active layers were $250 \mathrm{~nm}, 500 \mathrm{~nm}$ or $1 \mu \mathrm{m}$ thick and were grown at a rate of $\sim 1.2 \mu \mathrm{m} / \mathrm{h}$ with a substrate temperature of $490^{\circ} \mathrm{C}$, as measured by band edge thermometry (BET). Varying the $\mathrm{Tb}$ effusion cell temperature $\left(1100^{\circ} \mathrm{C}-1480^{\circ} \mathrm{C}\right)$ yielded atomic concentrations of $0.06 \%$ to $14 \%$ TbAs. Properties from two control samples, intrinsic InGaAs and InGaAs doped with Si to a level comparable to most of the TbAs:InGaAs samples $\left(2.5 \times 10^{18} \mathrm{~cm}^{-3}\right)$, are shown in each plot here as well.

TbAs growth rates were calibrated by Rutherford backscattering spectroscopy (RBS) measurements of previous samples, but RBS measurements of many of the current samples revealed slightly different concentrations, particularly at higher $\mathrm{Tb}$ cell temperatures where calibration samples were not available before. RBS was used for composition and thickness measurements instead of XRD because of both unclear XRD patterns caused by phase segregation in the samples and the inability to calculate concentration from changes in the lattice constants since they were super-dilated by the TbAs nanoparticles. ${ }^{6,10}$ RBS also revealed that the exact composition of the InGaAs varied up to $\pm 1 \%$ In and $\mathrm{Ga}$, owing to a slight instability in the flux from the In effusion cell. In most cases, samples with like TbAs concentrations behaved similarly, suggesting this variation does not significantly affect repeatability.

Three samples of varying TbAs concentrations were examined with high-angle annular dark field scanning transmission electron microscopy (HAADF STEM). Samples were first prepared by mechanical polishing (without $\mathrm{Ar}^{+}$ milling); then, plan-view micrographs were taken with an FEI Titan 80-300 STEM/TEM equipped with a fieldemission electron gun which was operated at $300 \mathrm{kV} .0 .8 \%$ and 14\% TbAs:InGaAs samples are shown in Fig. 1. With $0.8 \%$ TbAs, randomly dispersed nanoparticles can be seen which are cubic in shape with dimensions of $\sim 1 \mathrm{~nm}$ (as compared to $\sim 1.5 \mathrm{~nm}$ in $1.8 \% \mathrm{TbAs}: \mathrm{GaAs}$, which was grown at the same substrate temperature ${ }^{6}$ ); however, at the high concentration of $14 \%$, it is difficult to discern whether TbAs nanoparticles are much bigger or just overlap because of their large number. In ErAs nanocomposites, a concentration dependence was not found for nanoparticle size, but TbAs

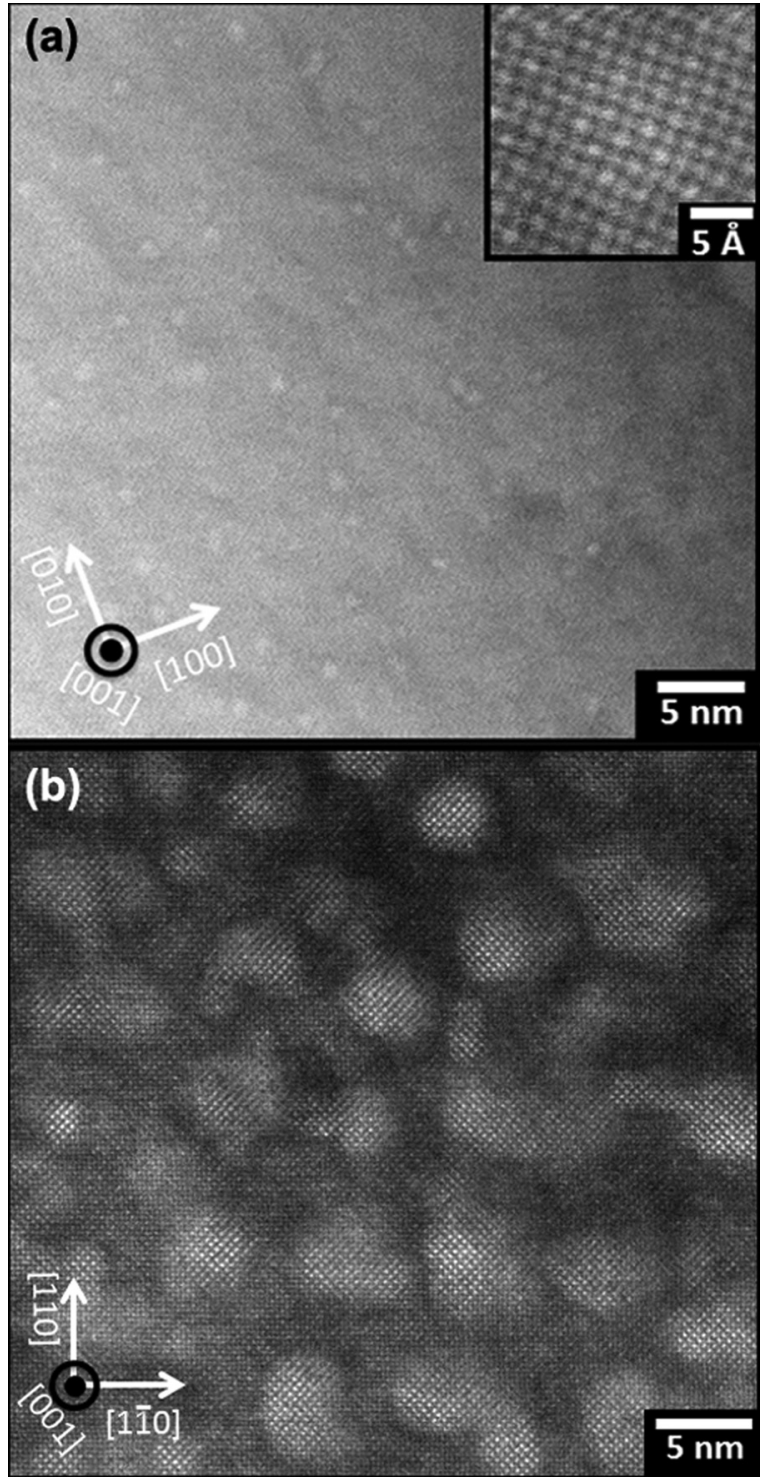

FIG. 1. (a) Randomly distributed TbAs nanoparticles of $\sim 1 \mathrm{~nm}$ in diameter were found in 0.8 at. \% TbAs:InGaAs with HAADF STEM while nanoparticles appear to be much larger and less uniform in size in 14 at. \% TbAs:InGaAs (b) possibly due to overlap of multiple particles.

nanocomposite growth conditions have not been explored deeply enough to extend this assumption. ${ }^{11}$ The rocksalt crystal structure of TbAs and the continuous As sublattice throughout the samples are also seen in these micrographs. On the other hand, nanoparticles were not observed by STEM in a $150 \mathrm{~nm} \times 150 \mathrm{~nm}$ area of a $0.2 \%$ TbAs sample (thickness unknown); this is contrary to STEM imaging of ErAs:InGaAs samples where nanoparticles were visible above $0.08 \%$ ErAs, raising the possibility that $\mathrm{Tb}$ is more soluble in InGaAs than Er. Nonetheless, it is impossible to rule out the existence of small clusters of TbAs at this low concentration that are not clearly visible with STEM.

\section{THERMOELECTRIC MEASUREMENTS AND DISCUSSION}

Resistivity and Hall effect measurements were taken for each sample using van der Pauw geometry on samples with 
indium contacts soldered at the corners. Conductivity, carrier concentration, and mobility were calculated from the results and are plotted versus TbAs concentration in Fig. 2. Similar trends and magnitudes of these values were observed in ErAs:InGaAs, but conductivity and carrier concentration peak at higher quantities of TbAs than of ErAs; indeed, conductivity peaks at $1 \%$ TbAs as opposed to $0.2 \%$ ErAs, and carrier concentration appears to peak at $3.3 \%$ TbAs instead of $1.2 \%$ in ErAs:InGaAs. ${ }^{12}$ Although it is apparent that $\mathrm{Tb}$ incorporation causes electrons to be donated to conduction since intrinsic InGaAs control samples were measured to have $\sim 2 \times 10^{16}$ n-type carriers, it is interesting to note that with more than $3.3 \% \mathrm{TbAs}$, carrier concentration actually decreases. This raises the question of how $\mathrm{Tb}$ atoms are arranged in higher concentration samples such that their electrons are not as available for conduction. Taking the STEM micrographs in Fig. 1 into account, it seems plausible that the ratio of $\mathrm{Tb}$ atoms within the nanoparticles to $\mathrm{Tb}$ atoms at the surface increases at higher concentrations (where the nanoparticles appear larger) and that core atoms do not donate electrons. This is surprising given that bulk TbAs is more conductive than many of these samples $\left(556 \Omega^{-1} \mathrm{~cm}^{-1}\right),{ }^{13}$ but strain on the TbAs nanoparticles from the InGaAs matrix and confinement effects could cause their band structure to change significantly, as was discussed in the Introduction. Hall effect measurements of two samples $(0.06 \%$ and $0.5 \%$, grown on the same day) are not reported due to a strong anisotropy between the two orthogonal in-plane

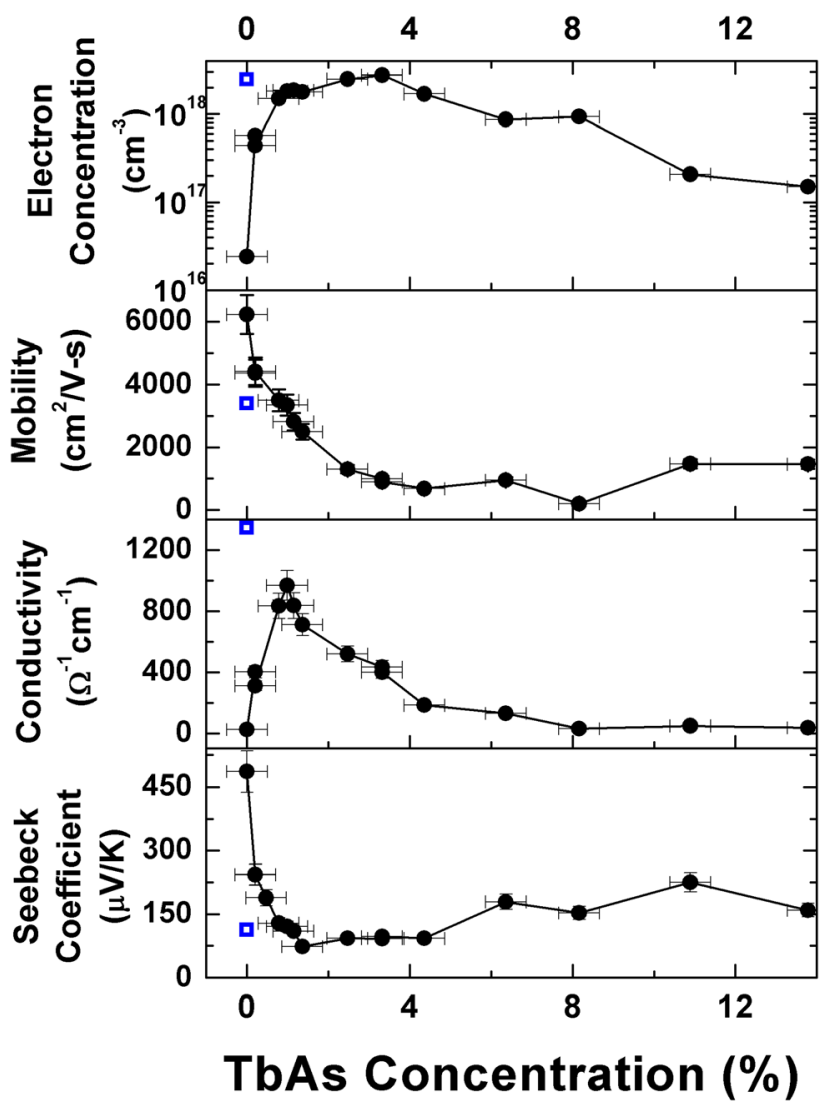

FIG. 2. Room temperature electron carrier concentration (a), electron mobility (b), electrical conductivity (c), and Seebeck coefficient (d) of TbAs:InGaAs are plotted as a function of TbAs concentration and compared with similarly doped Si:InGaAs (open square). directions - the cause of which is unclear-but all other samples were measurable and are reasonably isotropic.

Seebeck coefficient was measured at room temperature with a two point voltage measurement at either end of an approximately $5 \mathrm{~mm} \times 15 \mathrm{~mm}$ bar of sample material. Thermocouples at each end of the bar measured the temperature difference which was applied by two oppositely powered Peltier coolers upon which the ends of the bar rested. Fig. 2(d) shows that Seebeck coefficient loosely follows the inverse trend of carrier concentration and conductivity; this result is expected since as carrier concentration, and therefore, electrical conductivity, increase with electron donation from nanoparticles, the Fermi level moves up in the conduction band. This causes the differential electron conductivity to become more symmetric about the Fermi level (i.e., the moment is smaller), decreasing the Seebeck coefficient. ${ }^{14}$ Similarly, when carrier concentration falls after its peak around 3.3\% TbAs, Seebeck coefficient recovers somewhat for higher concentrations.

Thermal conductivity data were gathered using timedomain thermoreflectance (TDTR). ${ }^{15,16}$ Samples were prepared by cleaning the surface with acetone, isopropyl alcohol, deionized water, and a $40 \mathrm{~s}$ buffered hydrofluoric acid dip (in this order) and then depositing $60 \mathrm{~nm}$ of pure Al over the surface by e-beam evaporation. Fig. 3(a) shows that thermal conductivity decreases as more nanoparticles are introduced into the material with the exception of the first eight

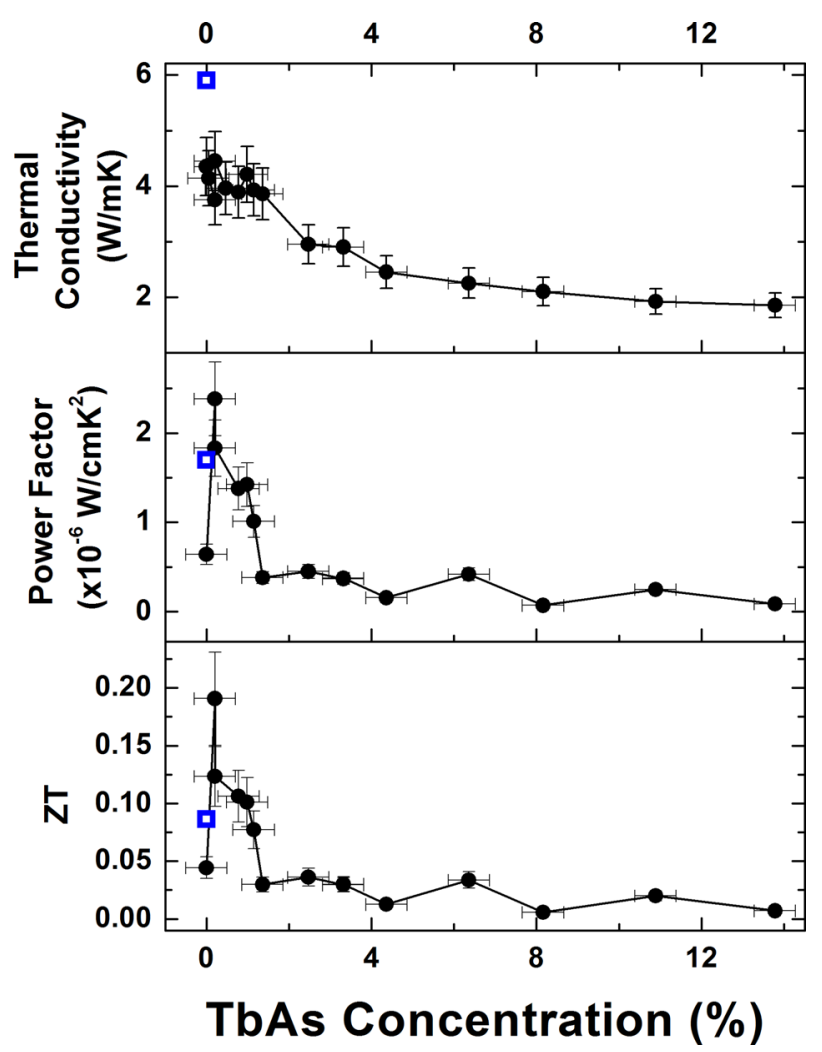

FIG. 3. Room temperature thermal conductivity (as measured by TDTR) (a), thermoelectric power factor (b), and thermoelectric figure of merit, ZT (c) as a function of TbAs nanoparticle concentration and compared to Si:InGaAs (open square). 
data points which stay around 3.5-4.5 W/mK-near the same value measured for intrinsic InGaAs thin films grown with this MBE system. For concentrations $>1.7 \%$, thermal conductivity is reduced significantly from that of the InGaAs control samples. STEM results described above revealing an apparent nanoparticle size dependence on $\mathrm{Tb}$ concentration could explain why thermal conductivity was not reduced much below that of the intrinsic InGaAs control samples at lower $\mathrm{Tb}$ concentrations; perhaps significant reduction of thermal conductivity requires a minimum nanoparticle size to efficiently scatter phonons. In comparison, unpublished TDTR data of low concentration ErAs:InGaAs samples $(0.08 \%-0.34 \%$ ErAs $)$ reveal thermal conductivity values $(4.89-4.36 \mathrm{~W} / \mathrm{mK})^{17}$ which are within the error bars of TbA$\mathrm{s}:$ InGaAs samples in the same concentration range. However, three-omega method thermal conductivity measurements of a different $0.3 \%$ ErAs:InGaAs sample returned a much lower value $(3.2 \mathrm{~W} / \mathrm{mK}){ }^{18}$

Thermoelectric power factor $\left(\mathrm{S}^{2} \sigma\right)$ and figure of merit, ZT $\left(\mathrm{S}^{2} \sigma \mathrm{T} / \kappa\right)$, were calculated for each sample and are shown in Figs. 3(b) and 3(c). The concentration at which both quantities are maximized at room temperature occurs around $0.2 \%$ TbAs. This does not necessarily imply that this concentration will perform best for high temperature applications. The maximum room temperature power factor $\left(2.38 \mathrm{~W} / \mathrm{mK}^{2}\right)$ and ZT $(0.19)$ recorded here are comparable to those observed in ErAs:InGaAs. The ErAs:InGaAs sample with the highest power factor $\left(2.96 \mathrm{~W} / \mathrm{mK}^{2}\right)$ and ZT $(0.20)$ also occurred near a concentration of $0.2 \%$ rare earth-arsenide. ${ }^{17,19}$ Since the thermal conductivity of $0.2 \%$ TbAs:InGaAs was not appreciably reduced below that of thin-film intrinsic InGaAs, it is clear that this sample performs best solely because of its increased power factor over both doped and undoped control samples. In particular, the balance of high Seebeck coefficient, which is more than twice that of Si-doped InGaAs, with electrical conductivity which is on the same order of magnitude as conventionally doped semiconductors, exemplifies the unique advantage of adding terbium to III-V semiconductors for more efficient thermoelectric power generation. On the other hand, the best-performing ErAs:InGaAs sample at room temperature $(0.2 \%$ ErAs) exhibited a $33 \%$ lower Seebeck coefficient and $173 \%$ higher electrical conductivity than $0.2 \% \mathrm{TbAs}$ and thermal conductivity that is comparable to $0.2 \% \mathrm{TbAs}$. The trade-off between Seebeck coefficient and electrical conductivity when switching from ErAs to TbAs doping is consistent with the predictions presented in the Introduction of this paper stating that the addition of strained TbAs nanoparticles (or small clusters) to InGaAs would pin the Fermi level below the conduction band, whereas the Fermi level of ErAs:InGaAs is above the conduction band minimum. Indeed, the energy band alignment of TbAs:InGaAs is expected to provide barriers for energy-dependent electron scattering, thereby increasing the Seebeck coefficient from ErAs:InGaAs. Even if at $0.2 \%$ TbAs, Tb atoms acts as single-ion dopants, the increase in Seebeck coefficient could conceivably be attributed to a variation in electric potential around a $\mathrm{Tb}$ ion or $\mathrm{TbAs}$ compound. Further, with a Fermi level placed within the conduction band, it is not surprising that ErAs:InGaAs is much more conductive than TbAs:InGaAs.

Perhaps the most interesting difference between the highest ZT TbAs:InGaAs and ErAs:InGaAs materials at room temperature would be that nanoparticles were observed in $0.2 \%$ ErAs:InGaAs by STEM but not in $0.2 \%$ TbAs:InGaAs, as was discussed earlier. Although measurements were recorded for ErAs:InGaAs samples where no nanoparticles were visible by STEM $(<0.08 \%$ ErAs $)$, these materials did not perform as well thermoelectrically. ${ }^{12,19}$ Although $\mathrm{Er}$ and $\mathrm{Tb}$ metals are very similar, and ErAs: InGaAs and TbAs:InGaAs tend to behave similarly as a function of rare earth-arsenide concentration, it seems that the physical and electrical methods by which TE performance with is best enhanced for these two systems are quite dissimilar.

\section{SUMMARY AND CONCLUSIONS}

The electrical and thermal transport properties of several TbAs:InGaAs nanocomposite thin films of varying TbAs concentration were measured to determine their potential for thermoelectric applications. Consistent with our expectations of improved thermoelectric performance due to the energy band alignment in this semimetal/semiconductor system, TbAs:InGaAs displays a large Seebeck coefficient with good electrical conductivity, leading to a room temperature ZT that is more than twice that of the control sample, silicondoped InGaAs. However, thermal conductivity was not appreciably reduced from that of intrinsic thin-film InGaAs except at TbAs concentrations above $1.7 \%$.

Trends in the room temperature thermoelectric measurements strongly parallel those found in ErAs:InGaAs nanocomposites, a thermoelectric material system that performed very well at high temperatures, leading us to expect similarly promising results from TbAs:InGaAs at high temperatures. Peak values in carrier concentration and electrical conductivity occurred at different concentrations for the two material systems. The properties defining the TbAs:InGaAs sample with the highest thermoelectric power factor and figure of merit differed from those of the ErAs:InGaAs material for which these quantities were maximized at room temperature. Although the best thermoelectric samples in both material systems contained $0.2 \%$ rare earth-arsenide, ZT was enhanced in $0.2 \%$ TbAs:InGaAs more so because of its higher Seebeck coefficient, whereas higher conductivity aided $0.2 \%$ ErAs:InGaAs. Moreover, no nanoparticles were visible in $0.2 \%$ TbAs:InGaAs with HAADF STEM while nanoparticles in $0.2 \%$ ErAs:InGaAs were clearly discernible. These distinctions highlight the electronic and physical differences between ErAs and the largely-unstudied TbAs, particularly when these semimetals are confined to small particles in varying strain environments, as well as their potential to lead to significant advancements in the thermoelectric power generation field by slightly different methods. Forthcoming measurements will determine these materials' thermoelectric performance at higher temperatures, and further improvement of efficiency will be sought in future materials grown using these results. 


\section{ACKNOWLEDGMENTS}

This project was funded at UD and UCSC by the Nanostructured Materials for Power program (DARPA-DSO, Army Research Office (Award No. W911NF0810347)) with support from the National Science Foundation IGERT at UD and at UCSB by the National Science Foundation through the UCSB MRL (Award No. DMR 05-20415) and the Army Research Office (Award No. W911NF-0701-0547). We thank Dr. Leszek Wielunski of the Rutgers University Tandem Accelerator Lab for collecting RBS data for these materials and Pernell Dongmo at the University of Delaware for assistance with measurement equipment and error estimation.

${ }^{1}$ G. J. Snyder and T. Ursell, Phys. Rev. Lett. 91, 148301 (2003).

${ }^{2}$ C. J. Vineis, A. Shakouri, A. Majumdar, and M. G. Kanatzidis, Adv. Mater. 22, 3970 (2010).

${ }^{3}$ W. Kim, J. Zide, A. Gossard, D. Klenov, S. Stemmer, A. Shakouri, and A. Majumdar, Phys. Rev. Lett. 96, 045901 (2006).

${ }^{4}$ J. M. Zide, D. O. Klenov, S. Stemmer, A. C. Gossard, G. Zeng, J. E. Bowers, D. Vashaee, and A. Shakouri, Appl. Phys. Lett. 87, 112102 (2005).

${ }^{5}$ J. M. O. Zide, J.-H. Bahk, R. Singh, M. Zebarjadi, G. Zeng, H. Lu, J. P. Feser, D. Xu, S. L. Singer, Z. X. Bian, A. Majumdar, J. E. Bowers, A. Shakouri, and A. C. Gossard, J. Appl. Phys. 108, 123702 (2010).
${ }^{6}$ L. E. Cassels, T. E. Buehl, P. G. Burke, C. J. Palmstrøm, A. C. Gossard, G. Pernot, A. Shakouri, C. R. Haughn, M. F. Doty, and J. M. O. Zide, J. Vac. Sci. Technol. B 29, 03 C114 (2011).

${ }^{7}$ K. Delaney, N. Spaldin, and C. Van De Walle, Phys. Rev. B 77, 235117 (2008).

${ }^{8}$ K. Delaney, private communication (April 2011).

${ }^{9}$ D. Vashaee and A. Shakouri, Phys. Rev. Lett. 92, 106103 (2004).

${ }^{10}$ M. A. Scarpulla, T. E. Buehl, B. Mellot, R. V. Chopdekar, K. M. Yu, R. Farshchi, and A. C. Gossard, presented at the North American Molecular Beam Epitaxy Conference, Breckenridge, Colorado, 2010.

${ }^{11}$ D. O. Klenov, J. M. O. Zide, J. M. LeBeau, A. C. Gossard, and S. Stemmer, Appl. Phys. Lett. 90, 121917 (2007).

${ }^{12}$ P. G. Burke, H. Lu, N. G. Rudawski, S. Stemmer, and A. C. Gossard, J. Vac. Sci. Technol. B 29, 03 C117 (2011).

${ }^{13}$ L. H. Brixner, J. Inorg. Nucl. Chem. 15, 199 (1960).

${ }^{14}$ J. Zide, D. Vashaee, Z. Bian, G. Zeng, J. Bowers, A. Shakouri, and A. Gossard, Phys. Rev. B 74, 205335 (2006).

${ }^{15}$ D. G. Cahill, W. K. Ford, K. E. Goodson, G. D. Mahan, A. Majumdar, H. J. Maris, R. Merlin, and S. R. Phillpot, J. Appl. Phys. 93, 793 (2003).

${ }^{16}$ G. Pernot, M. Stoffel, I. Savic, F. Pezzoli, P. Chen, G. Savelli, A. Jacquot, J. Schumann, U. Denker, I. Mönch, Ch. Deneke, O. G. Schmidt, J. M. Rampnoux, S. Wang, M. Plissonnier, A. Rastelli, S. Dilhaire, and N. Mingo, Nature Mater. 9, 491 (2010).

${ }^{17}$ P. G. Burke, private communication (2011).

${ }^{18}$ W. Kim, S. L. Singer, A. Majumdar, J. M. O. Zide, D. Klenov, A. C. Gossard, and S. Stemmer, Nano Lett. 8(7), 2097 (2008).

${ }^{19}$ H. Lu, P. G. Burke, A. C. Gossard, G. Zeng, A. T. Ramu, J.-H. Bahk, and J. E. Bowers, Adv. Mater. 23, 2377 (2011). 